\title{
Tempo ideal para a realização da visita domiciliar ao recém-nascido: uma revisão integrativa
}

\author{
Ideal time for home visits to newborns: an integrative review
}

\author{
Anniely Rodrigues Soares (https://orcid.org/0000-0002-3382-671X) ${ }^{1}$ \\ Anna Tereza Alves Guedes (https://orcid.org/0000-0002-9495-4942) ${ }^{2}$ \\ Tarciane Marinho Albuquerque de Vasconcelos Cruz (https://orcid.org/0000-0003-0780-117X) ${ }^{3}$ \\ Tayanne Kiev Carvalho Dias (https://orcid.org/0000-0002-6184-9281) ${ }^{2}$ \\ Neusa Collet (https://orcid.org/0000-0002-4795-0279) ${ }^{4}$ \\ Altamira Pereira da Silva Reichert (https://orcid.org/0000-0002-4295-6698) ${ }^{4}$
}

${ }^{1}$ Programa Institucional de Bolsas de Iniciação Científica, Faculdade de Enfermagem, Universidade Federal da Paraíba (UFPB). Campus I, Lot. Cidade Universitária. 58051-900 João Pessoa PB Brasil. anniely_rodrigues@ hotmail.com

${ }^{2}$ Programa de PósGraduação de Enfermagem, UFPB. João Pessoa PB Brasil.

${ }^{3}$ Programa de PósGraduação em Enfermagem, Centro de Ciências da Saúde, UFPB. João Pessoa PB Brasil.

${ }^{4}$ Departamento de Enfermagem de Saúde Pública e Psiquiatria, UFPB. João Pessoa PB Brasil.

\begin{abstract}
This study aimed to analyze in the scientific production in online journals regarding the ideal time to conduct home visits (HVs) to newborns (NBs) in their first week of life, and the difficulties in doing so due to the lack of a consensus around the subject. This is an integrative review based on data available on the MEDLINE, BVS, Web of Science and PubMed databases. Eight studies published between 2010 and 2015 were identified. The thematic analysis evidenced two topics, namely, the ideal time for the first home visit to the newborn, and difficulties in implementing the home visit to the newborn in the first week of life. The results suggest that the first visits should take place in the first week of life, especially within the second day after birth. However, some impediments to the realization of the home visit are found, such as geographical barrier, poor quality of care delivered by professionals, low number of notifications of mother-baby binomial hospital discharge, and mothers' lack of knowledge about the relevance of postnatal care. All this can impair the performance of health professionals, and requires investing in their professional qualification and service infrastructure.
\end{abstract}

Key words Newborn, Home Visit, Primary Health Care
Resumo O objetivo foi analisar a produção científica em periódicos online sobre o tempo ideal para a realização da visita domiciliar ao recémnascido, na primeira semana de vida, e as dificuldades para a efetivação desta, devido à ausência de um consenso acerca do tema. Trata-se de uma revisão integrativa que teve como fonte de dados publicações disponíveis nas bases MEDLINE, BVS, Web of Science e PubMed. Foram identificados 8 estudos publicados no período entre 2010 e 2015. A partir da análise temática evidenciaram-se duas áreas temáticas: Tempo ideal para a primeira visita domiciliar ao recém-nascido e Dificuldades para a efetivação da visita domiciliar ao recém-nascido na primeira semana de vida. Os resultados sugerem que as primeiras visitas devam ocorrer na primeira semana de vida, em especial, até o segundo dia após o nascimento. Porém, há barreiras para a concretização da visita domiciliar, como: barreira geográfica, baixa qualidade dos cuidados fornecidos pelos profissionais, escassa notificação da alta hospitalar do binômio mãe-bebê e desconhecimento das mães sobre a importância dos cuidados pós-natais. Tudo isso pode comprometer a atuação dos profissionais de saúde, fazendo-se necessário investir em capacitação destes e infraestrutura do serviço.

Palavras-chave Recém-nascido, Visita domiciliar, Atenção Primária à Saúde 


\section{Introdução}

O período neonatal, que compreende do nascimento até o $28^{\circ}$ dia de vida, é um momento considerado de vulnerabilidade para a saúde do RN. Portanto, os bebês necessitam de cuidados indispensáveis nos seus primeiros dias de vida, pois, anualmente, cerca de 2,6 milhões de RN morrem no primeiro mês de vida; sendo que aproximadamente 1 milhão destas mortes ocorrem nas primeiras 24 horas, e cerca de 1 milhão nos primeiros seis dias de vida. Além disso, projeta-se que 30 milhões de mortes infantis, entre 2017 e 2030, dar-se-ão no período neonatal ${ }^{1}$.

Diante desses índices preocupantes, faz-se notório que a atenção aos RNs influenciará na condição de vida e saúde do indivíduo até a fase adulta $^{2}$. Nessa perspectiva, estudo evidencia que $70 \%$ dos óbitos neonatais são considerados evitáveis, tendo como um dos principais motivos o fornecimento de cuidados ineficientes e negligentes pelos serviços de saúde ${ }^{3}$. Conquanto, graças aos esforços de países que buscam o progresso da saúde da criança, segundo o Relatório 2017 Níveis e Tendências em Mortalidade Infantil, o número de mortes de crianças menores de 5 anos foi reduzido de 9,9 milhões por ano, em 2000, para 5,6 milhões em 2016'. Em conformidade com o cenário mundial, o Brasil apresentou reduções anuais consecutivas da taxa de mortalidade infantil entre 1990 a 2015, todavia, este padrão de declínio foi rompido ao registrar um aumento de 14,3 mortes infantis para cada mil nascidos vivos, em 2015; para 14,9, em 2016 ${ }^{4}$. Desta forma, esse quantitativo precisa reduzir ainda mais, tendo em vista ser essa faixa etária em que há maior concentração da mortalidade infantil.

A fim de reduzir as taxas de mortalidade infantil, a Organização das Nações Unidas lançou em 2015 a "A agenda 2030, para o Desenvolvimento Sustentável", que tem como uma de suas finalidades dar continuidade aos progressos dos Objetivos de Desenvolvimento do Milênio e obter avanços nas metas não alcançadas. Essa agenda, constituída por 17 Objetivos de Desenvolvimento Sustentável e 169 metas, almeja em seu $3^{\circ}$ objetivo findar as mortes de RNs e crianças menores de 5 anos, por causas evitáveis, conduzindo a redução da mortalidade infantil no mundo ${ }^{5}$.

Quanto ao Brasil, a fim de prestar cuidados eficientes ao binômio puérpera-neonato, o serviço de saúde utiliza estratégias de influência significativa no bem-estar das pessoas, como a Visita Domiciliar (VD). Esta possui grande ênfase na Atenção Primária à Saúde (APS), por prestar, concomitantemente, um atendimento assisten- cial e educativo, além de fornecer cuidados que aumentam a sobrevivência neonatal. Portanto, a efetivação da VD pela equipe de saúde deve ser precoce, ocorrendo logo após o nascimento ${ }^{6}$.

A VD é uma prática de cuidado continuado em saúde no domicílio, constituída por elementos peculiares que dão seu significado. É uma atenção em saúde que visa a prevenção de doenças, promoção, tratamento e reabilitação da saúde do paciente e, além disso, é capaz de complementar e/ou substituir cuidados fornecidos em outros espaços. Assim, com essas características, a atenção domiciliar garante uma atenção educativa, humanizada, integral e resolutiva ${ }^{7}$.

Voltando-se o olhar para a positiva relação entre VD e promoção da saúde, o sistema de saúde brasileiro, recomenda que a atenção domiciliar deverá contemplar ações de promoção à saúde, prevenção e tratamento de doenças e reabilitação prestadas em domicílio, com garantia de continuidade de cuidados e integrada às Redes de Atenção à Saúde (RAS) .

Diante destes benefícios para saúde do indivíduo e, em particular, os ganhos para a saúde da criança, torna-se imprescindível a existência de visitas domiciliares voltadas para o neonato. Tendo em vista essa necessidade, o Ministério da Saúde aconselha sua efetivação na primeira semana de vida pós-parto².

A VD ao recém-nascido é uma ferramenta potente para prevenção de agravos e promoção de saúde que resulta em maior sobrevida neonatal ${ }^{9}$. Todavia, para o sucesso dessa estratégia, é necessário que sua efetivação seja no momento adequado e de maior vulnerabilidade para a saúde da criança.

São escassos os estudos e as organizações que determinam o tempo ideal para a realização dessa VD ao RN, porém, os estudos realizados evidenciaram que quando essas visitas são realizadas nas primeiras 48 horas após o parto, as intervenções pós-natais são capazes de prevenir mortes que acometem as crianças nesse perío$\mathrm{do}^{10,11}$. Essa intervenção se torna mais importante nos países em desenvolvimento, uma vez que os mesmos apresentam lacunas na assistência prénatal e no parto, representando riscos para o binômio mãe-bebê $\hat{e}^{12}$.

No Brasil, o Ministério da Saúde preconiza que a visita seja realizada no $5^{\circ}$ dia de vida da criança denominado o "5o Dia de Saúde Integral", no qual deverão ser realizadas as ações de saúde essenciais para o bebê e sua mãe no primeiro contato após a alta da maternidade ${ }^{13}$. Entretanto, estudo publicado no Brasil evidenciou situação preocupante em que a VD ao RN está sendo re- 
alizada após uma semana de vida, às vezes, em até 15 dias após a chegada da criança no seu domicílio ${ }^{14}$.

A Organização das Nações Unidas (ONU) e o Fundo das Nações Unidas para a Infância (UNICEF) no documento intitulado Home visits for the newborn child: a strategy to improve survival recomendam que a VD seja na primeira semana de vida, ao constatar que a maioria das mortes neonatais que ocorrem após as 48 horas de vida podem ser prevenidas por meio de cuidados imediatos ao neonato ${ }^{6}$.

Considerando que os primeiros dias de vida são determinantes para a sobrevivência do neonato, é fundamental que seja determinado o momento ideal, isto é, o momento exato para concretizar a VD e efetivar os cuidados de saúde iniciais e protetivos. Além disso, compreender quais são os principais fatores que dificultam a realização desta visita e buscar reduzi-los permitirá galgar novas conquistas para a saúde infantil.

Portanto, se justifica a relevância desta pesquisa para a população em geral e para a comunidade científica, bem como por ser um meio para atingir o bem-estar da tríade criança-mãe-família com intervenção em tempo hábil para prevenir os agravos que possam prejudicar a saúde da criança buscando, assim, promover seu bem-estar oportunamente. Deste modo, o presente estudo teve como objetivo analisar o tempo ideal para a realização da visita domiciliar ao recémnascido e as dificuldades para a efetivação desta nas produções científicas presentes na literatura nacional e internacional.

\section{Método}

Trata-se de um estudo de revisão integrativa da literatura, que teve por objetivo sintetizar os resultados de estudos sobre o tema ${ }^{15}$. A elaboração desse tipo de estudo envolveu várias etapas ${ }^{16}$. Inicialmente houve a elaboração das seguintes questões norteadoras: Qual o tempo ideal abordado na literatura para a realização da visita domiciliar ao RN, na primeira semana de vida? Quais as dificuldades para a efetivação da VD ao RN?

A busca na literatura foi realizada no período de outubro a dezembro de 2015, nas seguintes bibliotecas eletrônicas: Medical Literature Analysis and Retrieval System on-line (MEDLINE), PubMed, Biblioteca Virtual de Saúde (BVS) e Web of Science. Utilizou-se os descritores em português e inglês, dispostos segundo as padronizações dos Descritores em Ciências da Saúde (DECs) e Medical Subject Headings (MeSH), os quais foram:
Recém-Nascido ou Newborn; e Visita Domiciliar ou Home Visit. Esses descritores foram cruzados individualmente nas bibliotecas eletrônicas selecionadas, empregando o operador booleano " $A N D$ " de forma a garantir a inclusão de todos os artigos pertinentes à temática.

Foram considerados como critérios de inclusão: artigos publicados no período de 2010 a 2015; artigos completos disponíveis e indexados nas bibliotecas eletrônicas; publicações disponíveis nos idiomas inglês e português. Excluíram-se os estudos incompletos e que não atendiam ao tema proposto.

A busca totalizou um quantitativo de 1.646 artigos após filtragem inicial, obedecendo os critérios estabelecidos. Em seguida, os títulos e resumos foram criteriosamente lidos por dois pesquisadores separadamente, o que resultou em 430 estudos.

Foram extraídas as seguintes informações dos estudos selecionados: título, autores, ano de publicação, periódico, Journal Citation Report (JCR), qualis, fator de impacto, idioma, objetivo, o tempo ideal e as dificuldades para a realização da VD ao RN, período e local em que o estudo foi realizado. Justifica-se a escolha do Qualis e do indicador Journal Citation Reports (JCR) como dado para análise da qualidade dos estudos, pois permitem avaliar os principais periódicos de pesquisa do Brasil e do mundo, de acordo com sua classificação e o seu fator de impacto, respectivamente.

Após o registro, os dados foram sistematicamente revisados e, em seguida, interpretados e discutidos por meio da análise temática ${ }^{17}$. Tal processo contemplou três passos: a pré-análise, na qual foi estabelecido o primeiro contato com o material, com identificação das palavras-chaves; a exploração do material, por meio de recortes das partes representativas dos artigos incluídos na revisão; e o tratamento dos resultados obtidos e interpretações.

A partir da análise dos artigos foi possível construir duas áreas temáticas (AT): AT1- Tempo ideal para a primeira visita domiciliar ao recémnascido; AT2- Dificuldades para a efetivação da visita domiciliar ao recém-nascido na primeira semana de vida.

\section{Resultados e discussão}

Apenas 8 artigos estão entre os estudos selecionados que versam sobre o tempo ideal para a realização da VD ao RN, sendo cinco encontrados na PubMed, um na Web Of Science e dois na Bi- 
blioteca Virtual de Saúde, conforme apresentado no Quadro 1.

Neste estudo, o periódico selecionado na pesquisa com maior JCR, isto é, com maior relevância na área, foi o PLOS Medicine com 14.43. Diante deste dado, tem-se que esta revista serve como referência quando abordamos temas envolvendo a visita domiciliar ao RN no contexto da APS. Por sua vez, o periódico com menor JCR (0.83) foi o Public Health Nursing.

Além desses dados, foi possível observar, conforme Quadro 1, outros aspectos relevantes nos artigos selecionados, como: título, autores, ano, periódico, idioma, objetivo, tempo ideal para realização da VD, período e local em que o estudo foi realizado. A metade desses estudos foi publicada no ano de 2015, como demonstrado no Gráfico 1. Quanto ao idioma, constata-se que todos foram publicados em inglês. Em relação ao local do estudo, dois aconteceram na Tanzânia e os demais em locais diferentes como China, Uganda, Etiópia, Gana e Malawi.

\section{AT1- Tempo ideal para a primeira visita domiciliar ao recém-nascido}

A partir da análise dos estudos que compõem a AT1 foi possível detectar diversas indicações sobre o tempo ideal para a VD ao neonato. Assim, um estudo realizado em Uganda ${ }^{18}$ e outro em Bangladesh ${ }^{19}$, indicaram que o ideal seria a realização de três VD ao recém-nascido na primeira semana após o nascimento, por outro lado, estudos realizados na Tanzânia ${ }^{20,21}$ defendem a efetivação de duas VD nos primeiros dias de vida do bebê. Logo, foi recorrente nas pesquisas analisadas a indicação de que as primeiras visitas domiciliares ao $\mathrm{RN}$ ocorram durante a primeira semana de vida, no entanto, o mais aconselhável é que ocorra até o segundo dia após o nascimento ${ }^{10,20,21}$.

Um estudo de meta-análise com ensaios clínicos randomizados e quase-randomizados ${ }^{10}$, ao avaliar a importância da continuidade do cuidado para a redução da mortalidade perinatal, neonatal e materna, identificou a eficácia do atendimento domiciliar para a melhoria da saúde da criança e ressaltou a importância da realização desta visita ao recém-nascido entre 0-2 dias pós-parto. Nesta perspectiva, estudiosos padronizaram a efetivação da visita domiciliar por profissionais de saúde nos dias 1,3 e 7 de vida do RN e constataram os benefícios desta estratégia para a saúde neonatal e materna ${ }^{18}$.

Deste modo, o momento ideal para os cuidados de saúde direcionados ao RN no cenário domiciliar modifica-se de acordo com o local anali- sado, porém, a recomendação da efetivação precoce da visita domiciliar é compartilhada por todos os estudos analisados. Em Bangladesh, Malawi e Nepal foi implementado um programa de cuidados maternos e neonatais com visitas domiciliares até o terceiro dia após o parto. Apesar da divergência no número de mães e $\mathrm{RN}$ que receberam $\mathrm{o}$ atendimento nos diferentes países, recomenda-se a efetivação da visita domiciliar ao recém-nascido e à puérpera até o terceiro dia pós-natal, por meio dos sistemas comunitários de saúde eficazes ${ }^{19}$.

Cabe ressaltar que o prazo para a visita domiciliar até o segundo dia de vida inviabiliza o contato com o RN que nasceu em maternidade, tendo em vista que nesse período o bebê ainda se encontra na instituição hospitalar. Portanto, a indicação de VD ao RN no segundo dia de vida, nos estudos que recomendam a visita nesse período $^{20,21}$, provavelmente se deva ao fato do expressivo quantitativo de partos domiciliares e do não acesso das mulheres às instituições de saúde para o parto, resultando na principal causa de morbimortalidade materna e neonatal no país ${ }^{22}$.

Segundo o relatório Every child alive: the urgent need to end newborn deaths, os países mais perigosos de nascer e com as maiores taxas de morbimortalidade neonatal são os que a população apresenta baixa renda, como alguns localizados na África, que não dispõem de profissionais de saúde e parteiras treinadas que prestem assistência ao neonato e à puérpera com segurança ${ }^{23}$.

Assim sendo, os cuidados de saúde primários logo após o nascimento são fundamentais para a melhoria da saúde e redução da mortalidade neonatal ${ }^{24}$, principalmente em ambientes em que a população apresenta vulnerabilidade. Portanto, a efetivação da visita domiciliar pós-natal precoce é primordial para a orientação quanto às práticas de cuidados ao recém-nascido, como o aleitamento materno exclusivo, que devem ser promovidas tão logo o profissional tenha contato com o binômio mãe-bebê ${ }^{21}$.

Ademais, pesquisa realizada em diferentes regiões da Etiópia com alto índice de mortalidade infantil e baixa efetividade dos programas de saúde vigentes, identificou a necessidade de novos programas comunitários de saúde capazes de garantir o contato precoce entre profissionais de saúde e recém-nascidos através de estratégias de saúde como a visita domiciliar ${ }^{25}$.

Consoante ao exposto, a World Health Organization (WHO) também destaca a importância dos cuidados ao RN nas 48 horas após o parto, considerando ser este o momento crucial para a sobrevivência infantil, pois é um período em que ocorrem mais de $50 \%$ das mortes neonatais ${ }^{1}$. 


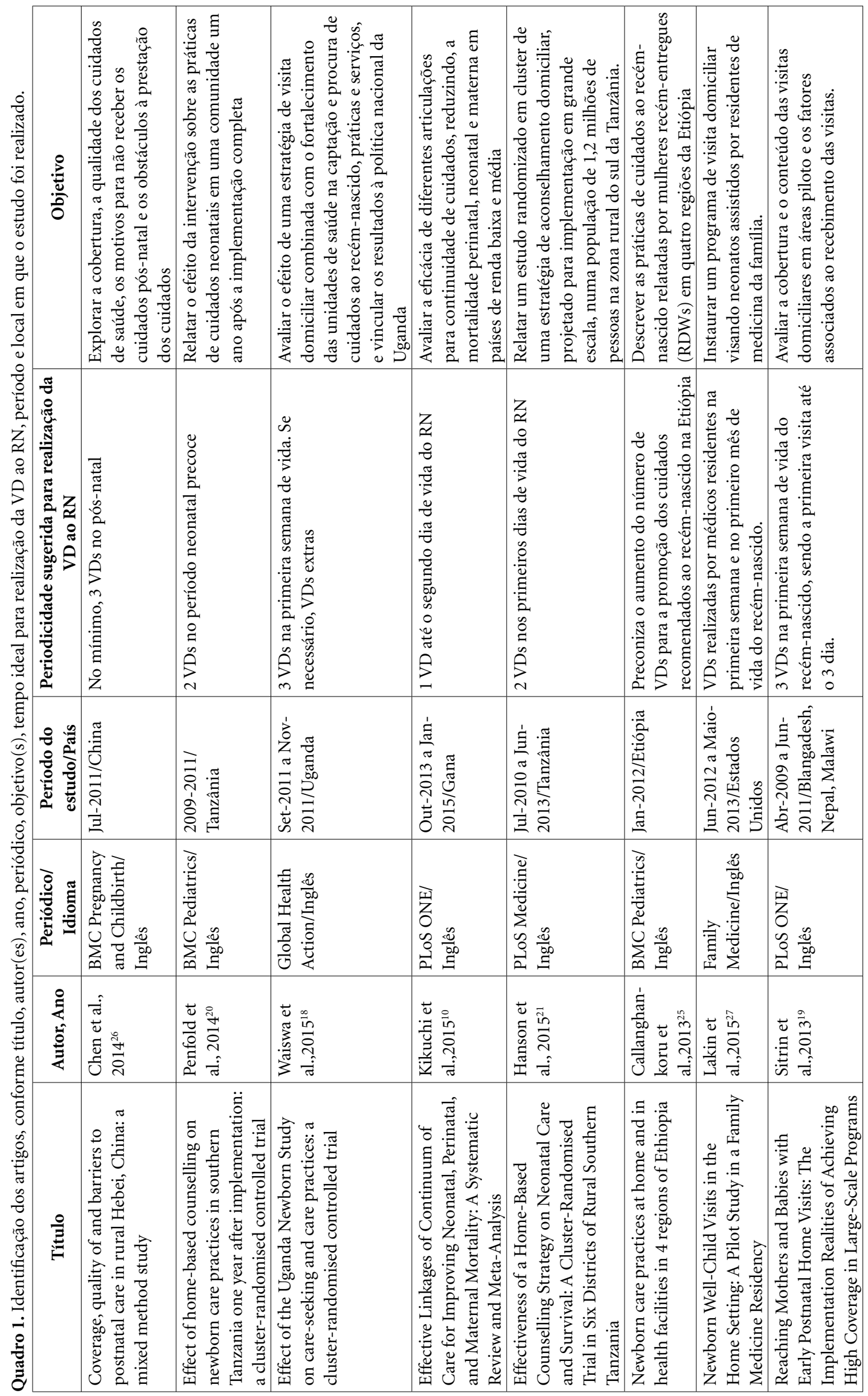




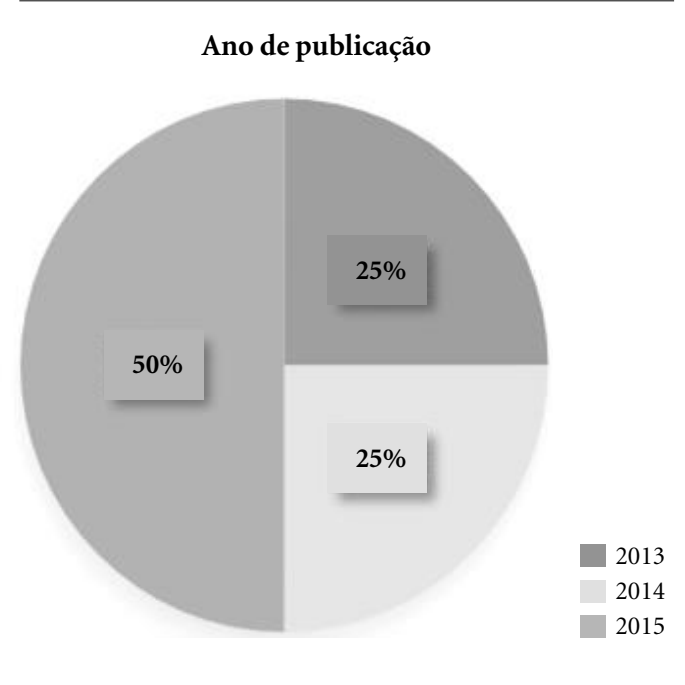

Gráfico 1. Distribuição da produção científica acerca do tempo ideal para a realização da visita domiciliar ao recém-nascido, segundo o ano de publicação.

Fonte: PIBIC, 2016.

Uma pesquisa que buscou avaliar o impacto de um treinamento de profissionais de saúde para a identificação precoce de modificações no estado geral do neonato, evidenciou que $2 / 3$ das crianças que receberam uma VD de um trabalhador de saúde capacitado, no dia do nascimento, tiveram maiores chances de sobreviver, devido à redução do risco de mortalidade neonatal. Além disso, também mostrou que a realização de uma visita no segundo dia de vida do neonato foi capaz de reduzir em mais de $50 \%$ o risco de morte entre as crianças que sobreviveram aos dois primeiros dias ${ }^{25}$.

Por outro lado, outros estudos ${ }^{20,21}$, apesar de indicar a efetivação precoce da primeira VD até o $2^{\circ}$ dia de vida, mostram índices diminutos de RN que receberam a visita neste período, $18 \%$ e $15 \%$, respectivamente. No estudo ${ }^{20}$ que determinou a realização de três visitas domiciliares durante a gestação e duas no período neonatal precoce, com visitas adicionais para neonatos prematuros, constatou que, apesar de encontrar uma associação positiva entre VD precoce e bem-estar neonatal, houve uma baixa adesão dos profissionais a esta determinação.

Um fator que pode influenciar no sucesso da realização da visita ao neonato nos dois primeiros dias de vida é o local onde ocorreu o parto, se no domicílio ou em uma instituição de saúde ${ }^{18}$. Isso foi constatado em estudo ${ }^{18}$ realizado na Uganda que concluiu que apenas $30 \%$ dos bebês nascidos que nasceram em instituição de saúde foram vi- sitados no segundo dia de vida. Diante desse resultado, infere-se que quando o parto ocorre em uma instituição de saúde, a intervenção domiciliar tende a ser adiada, não sendo possível realizá -la nos primeiros dias de vida do recém-nascido.

Por outro lado, pesquisa randomizada implementada em Uganda ${ }^{18}$ e com apoio da Organização Mundial de Saúde através da diretriz intitulada Joint Statement on home visits for the newborn child: a strategy to improve survival ${ }^{6}$ preconizam que a primeira visita domiciliar ao recém-nascido ocorra no primeiro dia de vida, e sejam feitas mais duas visitas, no $3^{\circ}$ e $7^{\circ}$ dia.

Contudo, ao analisar os artigos que compõem a presente revisão, e outras pesquisas que tratam da temática, viu-se que não há um consenso quanto ao momento ideal para a VD ao RN. No entanto, todos ressaltam a necessidade desta visita ocorrer o mais precocemente possível.

Mesmo diante da falta de consenso da literatura quanto ao tempo ideal, recomenda-se que a VD seja realizada por um profissional qualificado, para que este forneça atenção em saúde logo após o nascimento, independente do local do parto ${ }^{6}$. Por fim, o estabelecimento do momento mais oportuno para a efetivação da VD ao RN trará benefícios imensuráveis para a saúde da criança, todavia, para a concretização desta intervenção também se faz necessário o controle das dificuldades enfrentadas, a partir do empenho de todos os profissionais da Rede de Atenção à Saúde envolvidos com o cuidado ao recém-nascido.

\section{AT2- Dificuldades para a efetivação da visita domiciliar ao recém-nascido na primeira semana de vida}

Para que a visita domiciliar alcance o seu objetivo positivo para a saúde do recém-nascido, é importante aptidão e disponibilidade do profissional de saúde para realizá-la como também, que os serviços de saúde forneçam condições favoráveis para o encontro da mãe com o profissional ${ }^{26}$. Assim, a VD é condicionada por estes e outros fatores que determinam sua realização, ou não, podendo considerá-los como empecilhos para a sobrevida neonatal, quando não permitem a atenção domiciliar de qualidade.

Consoante aos estudos da AT2 (Quadro 2), a baixa qualidade dos cuidados fornecidos pelos profissionais de saúde aos recém-nascidos e a ausência de transporte para solucionar o problema da distância geográfica, foram as dificuldades mais recorrentes que interferem na concretização da VD (Gráfico 2), segundo estudos analisa$\operatorname{dos}^{19,23}$. 
Quadro 2. Artigos da amostra que compõem a área temática 2.

\begin{tabular}{|c|c|}
\hline \multicolumn{2}{|r|}{ AT2 - Dificuldades para a efetivação da visita domiciliar ao recém-nascido na primeira semana de vida } \\
\hline $\begin{array}{c}\text { Código } \\
\text { identificador }\end{array}$ & Título dos artigos \\
\hline AT2a & Newborn care practices at home and in health facilities in 4 regions of Ethiopia \\
\hline AT2b & $\begin{array}{l}\text { Effect of home-based counselling on newborn care practices in southern Tanzania one year } \\
\text { after implementation: a cluster-randomised controlled trial }\end{array}$ \\
\hline AT2c & Newborn Well-Child Visits in the Home Setting: A Pilot Study in a Family Medicine Residency \\
\hline AT2d & $\begin{array}{l}\text { Effectiveness of a Home-Based Counselling Strategy on Neonatal Care and Survival: A Cluster- } \\
\text { Randomised Trial in Six Districts of Rural Southern Tanzania }\end{array}$ \\
\hline AT2e & $\begin{array}{l}\text { Effect of the Uganda Newborn Study on care-seeking and care practices: a cluster-randomised } \\
\text { controlled trial }\end{array}$ \\
\hline AT2f & Coverage, quality of and barriers to postnatal care in rural Hebei, China: a mixed method study \\
\hline AT2g & $\begin{array}{l}\text { Reaching Mothers and Babies with Early Postnatal Home Visits: The Implementation Realities } \\
\text { of Achieving High Coverage in Large-Scale Programs }\end{array}$ \\
\hline
\end{tabular}

Fonte: PIBIC, 2016.

\section{As dificuldades para a efetivação e para o acolhimento da visita domiciliar ao recém-nascido} e sua frequência nos artigos analisados

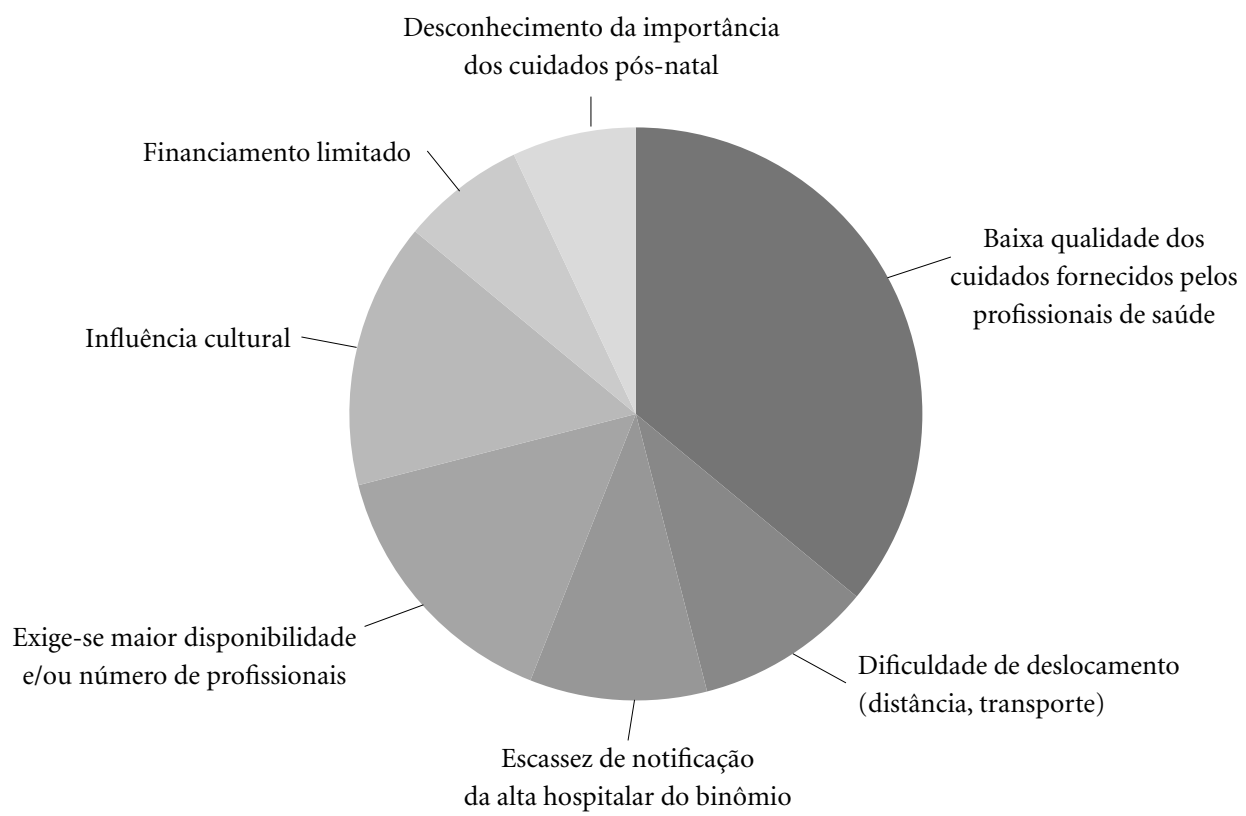

Gráfico 2. Distribuição da produção científica acerca das dificuldades para a efetivação e acolhimento da visita domiciliar ao recém-nascido.

Fonte: PIBIC, 2016.

Estudo randomizado desenvolvido em áreas rurais da Tanzânia ${ }^{21}$, onde 824 voluntários foram treinados para aconselhar às mães e familiares, por meio de visitas domiciliares, acerca dos cuidados necessários com o neonato e analisar a so- brevivência neonatal nesta população, evidenciou diversas barreiras que podem provocar a baixa cobertura de cuidados pós-parto. A principal delas foi o longo percurso percorrido para ter contato com o binômio mãe-criança, além da baixa qua- 
lidade dos serviços prestados nas instalações de saúde ${ }^{21}$. Uma dificuldade semelhante encontrada foi o longo tempo necessário para o deslocamento dos profissionais de saúde até a residência do RN em estudo realizado nos Estados Unidos ${ }^{27}$.

Voltando-se para o contexto da China Rural, pesquisadores destacaram como dificuldades para a realização da visita domiciliar ao recémnascido: a influência cultural, nível de conhecimento materno reduzido sobre a importância dos cuidados pós-natal, quantitativo reduzido de profissionais de saúde para atender ao binômio mãe -bebê, financiamento governamental inadequado para a saúde e ausência de transporte para o deslocamento dos profissionais até os domicílios ${ }^{26}$.

A partir dos artigos mencionados, constatase que, apesar de os estudos serem realizados em países com culturas diferentes, uma das dificuldades mais comuns entre eles para a realização efetiva da visita domiciliar ao recém-nascido é a barreira geográfica, ou seja, a distância entre a unidade de saúde e o domicílio.

Um estudo com 1.601 cuidadores de crianças menores de dois anos e profissionais de saúde, a fim de quantificar a cobertura dos cuidados fornecidos aos bebês, analisar a sua qualidade e identificar os motivos para a não realização deste, constatou que a escassez de profissionais, o treinamento inadequado dos mesmos e a ausência de transporte interferem na qualidade das visitas domiciliares realizadas ao binômio mãe-bebê ${ }^{26}$.

$\mathrm{O}$ estudo realizado na Etiópia ${ }^{25}$ destacou que as mulheres reduziram a procura por serviços de saúde ao perceber baixa qualidade dos cuidados fornecidos pelos profissionais elas e aos seus filhos. Portanto, atentar para esta realidade é de suma importância, pois a má qualidade do cuidado em saúde, além de impedir ações preventivas e de promoção da saúde como a VD, pode gerar um problema de saúde pública com proporções ainda maiores para a população em geral.

A fim de sanar essa fragilidade, a lei brasileira $\mathrm{n}^{\mathrm{o}} 13.257$ de 8 de março de $2016^{28}$ garante que os programas de VD voltados ao cuidado e educação na primeira infância deverão contar com profissionais qualificados, apoiados por medidas que assegurem sua permanência e formação continuada.

Outra adversidade preponderante encontrada foi a escassa notificação da alta hospitalar do binômio mãe-neonato, aumentando a dificuldade do profissional de saúde em assistir precocemente o recém-nascido, no qual o atraso da comunicação sobre a alta da mãe e criança teve grande influência na baixa cobertura de cuidados pós-parto ${ }^{21}$.
Em Uganda, um estudo evidenciou que, mesmo que as mulheres tenham recebido alta precoce, em 24 horas após o parto, os Agentes Comunitários de Saúde (ACSs) as visitaram mais tarde do que as mulheres que deram à luz em casa. Isto ocorreu devido, possivelmente, à falta de notificação da alta do binômio mãe-bebê ${ }^{18}$.

No Brasil, é primordial a contra referência sistemática e formalizada do recém-nascido da atenção hospitalar para a Estratégia Saúde da Família, pois a comunicação eficiente entre os níveis de atenção à saúde favorece o compartilhamento de casos, a atuação precoce do profissional de saúde e a continuidade da atenção ao binômio neonato-puérpera, principalmente na atenção básica ${ }^{29}$.

Considerando que, no cenário brasileiro, a porcentagem de nascidos vivos de parto hospitalar aumentou de $96,5 \%$ em 2000 para 98,4\% em 2015, pressupõe-se que, após a alta hospitalar, o número de recém-nascidos que necessitam dos cuidados de saúde na atenção básica também é crescente no país. Assim, a efetiva comunicação entre a atenção primária, secundária e terciária facilitará o processo de trabalho dos profissionais de saúde para a identificação precoce dos usuários ${ }^{30}$.

Sendo assim, faz-se necessária a adoção de estratégias para envolver a família e a comunidade nos locais onde é difícil a identificação de gestantes pelos ACS. Por exemplo, criação de grupos de mulheres na comunidade para facilitar o acesso do ACS às informações acerca da alta hospitalar do binômio puérpera-neonato, favorecendo, assim, visita de rotina precoce desses profissionais ${ }^{19}$.

A ausência de conhecimento das puérperas sobre a importância dos cuidados pós-parto para a saúde materna e do RN se apresenta como outra dificuldade para a efetivação da $\mathrm{VD}^{26}$. Estudo realizado na $\mathrm{China}^{18}$ reafirma a necessidade de esclarecer às mulheres quanto à importância dos cuidados puerperais e neonatais, e do seu papel preventivo e promotor de saúde para a dupla mãe-bebê. Desta forma, o desconhecimento das mães não será mais uma dificuldade para a $\mathrm{VD}$, pois, sabendo da importância da VD no período pós-parto, as puérperas provavelmente não oferecerão resistência para a sua realização.

Ademais, outra dificuldade para a efetivação da VD precoce diz respeito à realidade brasileira de nascimentos por cesarianas, isso porque, $o$ Brasil apresenta a segunda maior taxa de cesáreas do mundo, com cerca de $55,6 \%$, o que impede que a mãe não esteja no segundo dia de vida em casa $^{31}$. No entanto, nos últimos anos, percebe-se um movimento brasileiro de resgate do parto humanizado, assim como, a procura das mulheres pelo parto domiciliar planejado ${ }^{32}$, o que exigirá 
dos profissionais de saúde a efetivação da VD precoce ao recém-nascido.

Diante do exposto, apreende-se que ao reduzir os empecilhos para a efetivação da VD na primeira semana de vida, será possível um cuidado com qualidade e no momento oportuno em que o neonato necessita de maior atenção, isto é, no tempo ideal para a VD ao RN que deverá ocorrer nos seus primeiros dias de vida.

\section{Conclusão}

A adoção de estratégias e planos para reduzir a mortalidade infantil, principalmente a neonatal, é uma ação realizada pela maioria dos países do mundo. Para isso, estudos confirmam que um instrumento de grande valor e de aceitabilidade no âmbito da saúde é a Visita Domiciliar ao recém-nascido.

Em conformidade com os artigos que constituem a Área Temática 1, três visitas domiciliares na primeira semana de vida da criança são necessárias para trazer benefícios para a saúde do neonato e o tempo ideal para esta intervenção. Além disso, recomenda-se que o primeiro contato do profissional de saúde com a criança durante a VD ocorra entre o nascimento e o segundo dia de vida da criança.

Por sua vez, os estudos que formam a Área Temática 2 identificaram que as principais difi-

\section{Colaboradores}

AR Soares: concepção do estudo, coleta de dados, análise e interpretação dos dados e redação do artigo. ATA Guedes e TKC Dias: análise e interpretação dos dados e redação do artigo. TMAV Cruz: coleta de dados, análise e interpretação dos dados, redação do artigo e revisão crítica. $\mathrm{N} \mathrm{Col-}$ let e APS Reichert: concepção do estudo, revisão crítica e aprovação da versão a ser publicada.

\section{Agradecimentos}

Este trabalho foi apoiado pelo Conselho Nacional de Desenvolvimento Científico e Tecnológico (CNPq) e pela Coordenação de Aperfeiçoamento de Pessoal de Nível Superior (CAPES), mediante a concessão de bolsas de Iniciação Científica à ATA Guedes e AR Soares. culdades para a realização da VD na primeira semana são: dificuldade no deslocamento (distância, falta de transporte), maior carga de trabalho dos profissionais de saúde, escassez na notificação da alta hospitalar do binômio puérpera-neonato, financiamento limitado, influência cultural, baixa qualidade dos cuidados fornecidos pelos profissionais ao recém-nascido e ausência de conhecimento materno sobre a importância dos cuidados pós-natais.

Diante desses resultados, torna-se inquestionável a relevância desse estudo para redirecionar o olhar dos gestores e profissionais de saúde e da comunidade acadêmica em favor da visita domiciliar precoce ao recém-nascido e buscar soluções para sanar as dificuldades existentes. Ademais, estudos avaliativos são recomendados para investigar a efetividade da visita domiciliar na primeira semana de vida para a redução da morbimortalidade infantil. Destaca-se como limitação do presente estudo o reduzido número de artigos internacionais acerca da temática e ausência de estudo nacional, o que dificultou visualizar a realidade brasileira. Além disso, a maioria dos estudos analisados aconteceu em países onde é expressivo o número de partos fora das instituições de saúde e sem o acompanhamento de profissionais treinados. Dessa forma, a indicação da VD ao RN no segundo dia de vida é questionável quando o parto ocorrer no contexto institucional. Ressaltase também como limitação do estudo a ausência da seleção de artigos no idioma espanhol.

\section{Referências}

1. World Health Organization (WHO). United Nations Children's Fund. Levels \& Trends in Child Mortality. Genebra: WHO; 2017.

2. Brasil. Ministério da Saúde (MS). Secretaria de Atenção à Saúde. Departamento de Ações Programáticas e Estratégicas. Atenção à saúde do recém-nascido: Guia para os profissionais de saúde. 2a ed. Brasília: MS; 2012.

3. Gaiva MAM, Fujimorin E, Sato APS. Mortalidade neonatal: análise das causas evitáveis. Rev Enferm UERJ 2015; 23(2):247-253.

4. Brasil. Ministério da Saúde (MS). Observatório da criança e do adolescente. Taxa de mortalidade na infância [Internet]. [acessado 2018 Set 01]. Disponível em: https:// observatoriocrianca.org.br/cenario-infancia/temas/ sobrevivencia-infantil-infancia/619-taxa- de-mortalidade-na-infancia-para-1-000-nascidos-vivos?filters=1,233

5. Brasil. Organização das Nações Unidas (ONU). Transformando nosso mundo: a agenda 2030 para o desenvolvimento sustentável. Rio de Janeiro: ONU; 2015. 
6. World Health Organization (WHO). United Nations Children's Fund. Join statement on home visits for the newborn child: a strategy to improve survival. Genebra: WHO; 2009.

7. Brasil. Ministério da Saúde (MS). Caderno de atenção domiciliar. Melhor em casa: a segurança do hospital no conforto do seu lar. Brasília: MS; 2012.

8. Brasil. Portaria no 963, de 27 de maio de 2013. Redefine a Atenção Domiciliar no âmbito do Sistema Único de Saúde (SUS). Diário Oficial da União 2013; 27 mai.

9. Martins RMG. Acompanhamento à puérpera e ao recém-nascido por meio de protocolo [especialização]. Minas Gerais: Universidade Federal de Minas Gerais; 2013.

10. Kikuchi K, Ansah EK, Okawa S, Enuameh Y, Yasuoka J, Nanishi K, Shibanuma A, Gyapong M, Owusu-agyei S, Oduro AR, Asare GQ, Hodgson A, Jimba M. Effective Linkages of Continuum of Care for Improving Neonatal, Perinatal, and Maternal Mortality: A Systematic Review and Meta-Analysis. Rev Plos One 2015; 10(9):1-27.

11. World Health Organization (WHO). WHO recommendations on postnatal care of the mother and newborn. Geneva: WHO; 2013.

12. Viellas EF, Domingues RMSM, Dias MAB, Gama SGN, Theme Filha MM, Costa IV, Bastos MH, Leal MC. Prenatal care in Brazil. Cad Saúde Pública 2014; 30(Supl. 1):S1-S15.

13. Brasil. Ministério da Saúde (MS). Secretaria de Atenção à Saúde. Departamento de Ações Programáticas Estratégicas. Política Nacional de Atenção Integral à Saúde da Criança: orientações para implementação. Brasília: MS; 2018.

14. Lucena DBA, Guedes ATA, Cruz TMAV, Santos NCCB, Collet N, Reichert APS. Primeira semana saúde integral do recém-nascido: ações de enfermeiros da Estratégia Saúde da Família. Rev Gaúcha Enferm 2018; 39:e2017-0068.

15. Ercole FF, Melo LS, Alcoforado CLGC. Revisão integrativa versus revisão sistemática. Rev Min Enferm 2014; 18(1):12-14.

16. Mendes KDS, Silveira RCCP, Galvão CM. Revisão integrativa: método de pesquisa para a incorporação de evidências na saúde e na enfermagem. Texto Contexto Enferm 2008; 17(4):758-764.

17. Minayo MCS. O desafio do conhecimento: pesquisa qualitativa em saúde. São Paulo: Hucitec; 2016.

18. Waiswa P, Pariyo G, Kallander K, Akuze J, Namazzi G, Ekirapa-kiracho E, Kerber K, Sengendo H, Aliganyira P, Lawn JE, Pertenson S. Effect of the Uganda Newborn Study on care-seeking and care practices: a cluster-randomised controlled trial. Rev Glob Health Action. Ghana 2015; 8:1-11.

19. Sitrin D, Guenther T, Murray J, Pilgrim N, Rubayet S, Ligowe R, Pun B, Malla H, Moran A. Reaching Mothers and Babies with Early Postnatal Home Visits: The Implementation Realities of Achieving High Coverage in Large-Scale Programs. Plos One 2013; 8(7):1-9.

20. Penfold S, Manzi F, Mkumbo E, Temu S, Jaribu J, Shamba D, Mshinda H, Cousens S, Marchant T, Tanner M, Schellenberg D, Schellenberg JA. Effect of home-based counselling on newborn care practices in southern Tanzania one year after implementation: a cluster-randomised controlled trial. BMC Pediatr 2014; 14(187):1-12.
21. Hanson C, Manzi F, Mkumbo E, Shirima K, Penfold S, Hill Z, Shamba D, Jaribu J, Hamisi Y, Soremekun S, Cousens S, Marchant T, Mshinda H, Schellenberg D, Tanner M, Schellenberg JA. Effectiveness of a Home-Based Counselling Strategy on Neonatal Care and Survival: A Cluster-Randomised Trial in Six Districts of Rural Southern Tanzania. Plos Med 2015; 12(9):122.

22. Ministry of Health and Social Welfare (MoHSW). Tanzania Health Sector Strategic Plan July 2015 - June 2020 (HSSP IV). 2015.

23. United Nations Children's Fund (UNICEF). Every child alive: the urgent need to end newborn deaths. Nova York: UNICEF; 2018.

24. Sacks E, Freeman PA, Sakyi K, Jennings MC, Rassekh BM, Gupta S, Perry HB. Comprehensive review of the evidence regarding the effectiveness of communitybased primary health care in improving maternal, neonatal and child health: 3 . neonatal health findings. $J$ Global Health 2017; 7(1):1-12.

25. Callanghan-koru JA, Seifu A, Tholandi M, Graftjohnson J, Daniel E, Rawlins B, Worku B, Baqui AH. Newborn care practices at home and in health facilities in 4 regions of Ethiopia. BMC Pediatrics 2013; 13(198):1-11.

26. Chen L, Qiong W, Velthoven MHV, Yanfeng Z, Shuyi Z, Ye L, Wei W, Xiaozhen D, Ting Z. Coverage, quality of and barriers to postnatal care in rural Hebei, China: a mixed method study. BMC Pregnancy Childbirth 2014; 14(31):1-12.

27. Lakin A, Sutter MB, Magee S. Newborn well-child visits in the home setting: A pilot study in a Family Medicine Residency. Rev Family Med 2015; 47(3):217221.

28. Brasil. Lei no 13.257, de 8 de março de 2016. Dispõe sobre as políticas públicas para a primeira infância. Diário Oficial da União 2016; 8 mar.

29. Sassá AH, Rosa TCS, Souza SNDH, Rosetto EG. Visitas domiciliares como instrumento na assistência ao recém-nascido de muito baixo peso e sua família. Rev Cien Cuid Saúde 2011; 10(4):713-721.

30. Fundação Oswaldo Cruz (Fiocruz). Avaliação do desempenho do Sistema de Saúde - Partos hospitalares [Internet]. [acessado 2017 Dez 10]. Disponível em: http://www.proadess.icict.fiocruz.br/index.php?p=1 \&pag=fic\&cod $=$ A10\&tab $=1$

31. World Health Organization (WHO). WHO recommendations: intrapartum care for a positive childbirth experience. Genebra: WHO; 2018.

32. Koettker JG, Bruggemann OM, Knobel R. Resultados maternos dos partos domiciliares planejados assistidos por enfermeiras da equipe Hanami no sul do Brasil. Texto Contexto Enferm 2017; 26(1):1-11.
Artigo apresentado em 14/12/2017

Aprovado em 22/10/2018

Versão final apresentada em 24/10/2018 